\title{
Malignant Peripheral Nerve Sheath Tumor in Neurofibromatosis Type I : Unusual Presentation of Intraabdominal or Intrathoracic Mass
}

\author{
Jong Gwang Kim, M.D., Woo Jin Sung, M.D., Dong Hwan Kim, M.D., \\ Young Hwan Kim, M.D. ${ }^{2}$, Sang Kyun Sohn, M.D. and Kyu Bo Lee, M.D. \\ Departments of Oncology/Hematology and Diagnostic Radiology? \\ Kyungpook National University Hospital, Daegu, Korea
}

\begin{abstract}
A malignant peripheral nerve sheath tumor (MPNST) is an extremely rare soft tissue tumor in the general population. On the other hand, there is a higher incidence of MPNST in patients with neurofibromatosis type I (von Recklinghausen's disease). The common sites are the extremities, trunk, head and neck. However, an intraabdominal or intrathoracic manifestation is uncommon. This paper reports two patients, a 31 year-old woman with multiple neurofibromatosis presenting as an intraabdominal malignant peripheral nerve sheath tumor, and a 33 year-old woman with an intrathoracic malignant peripheral nerve sheath tumor. The patients were treated with chemotherapy followed by radiotherapy. However, one patient died as a result of disease progression 21 months after the diagnosis and the other patient is currently being treated with radiotherapy.
\end{abstract}

Key Words : Neurofibromatosis, Sarcoma, Chemotherapy

\section{INTRODUCTION}

A malignant peripheral nerve sheath tumor (MPNST) is an extremely rare soft tissue tumor ${ }^{1)}$, whereas there is a higher incidence in patients with neurofibromatosis type I (NF1, von Recklinghausen's disease) $)^{2,3)}$. The prognosis of a MPNST associated with neurofibromatosis is generally worse than that without neurofibromatosis. The common sites of invasion are the lower and upper extremities, trunk, head and neck"). However, an intraabdominal or intrathoracic manifestation is rare. We recently encountered two cases of an intraabdominal or intrathoracic presentation of a MPNST in patients with neurofibromatosis.

\section{CASE REPORT}

Case I : A 31 year-old woman with a known neurofibromatosis was admitted complaining of a painful abdominal mass. A physical examination revealed a large, hard soft-tissue mass bulging out from the middle abdomen, and numerous, small subcutaneous neurofibroma nodules and brownish-pigmented lesions (café au lait spots) in the trunk and extremities (Figure 1). An abdominal CT scan demonstrated a large (approximately $9 \times 10 \mathrm{~cm}$ ) inhomogeneous mass with an area of central necrosis (Figure 2). The histopathology of the circumscribed and encapsulated mass obtained by a wide excision indicated a MPNST from the mesentery showing frequent mitoses in more than five per one high power field (Figure 3A). In the immunohistochemical stain, the tumor cells stained positively to the S-100 protein, which is a cytoplasmic marker used for identifying Schwann cells (Figure 3B). One month after surgery, the masses of the right paracolic gutter and the left supravesicle area appeared in the CT scan. The patient was then treated with 6 cycles of MAID chemotherapy (ifosfamide $2000 \mathrm{mg} / \mathrm{m}^{2}$ for 3 days, doxorubicin $20 \mathrm{mg} / \mathrm{m}^{2}$ for 3 days, and

- Received : January 26, 2004

- Accepted : April 16, 2004

- Correspondence to : Sang Kyun Sohn, M.D., Department of Oncology/Hematology, Kyungpook National University Hospital, 50 Samduck 2-Ga, Jung-Gu, Daegu, 700-721, Korea Tel : 82-53-420-5587, Fax : 82-53-426-2046, E-mail : sksohn@knu.ac.kr 


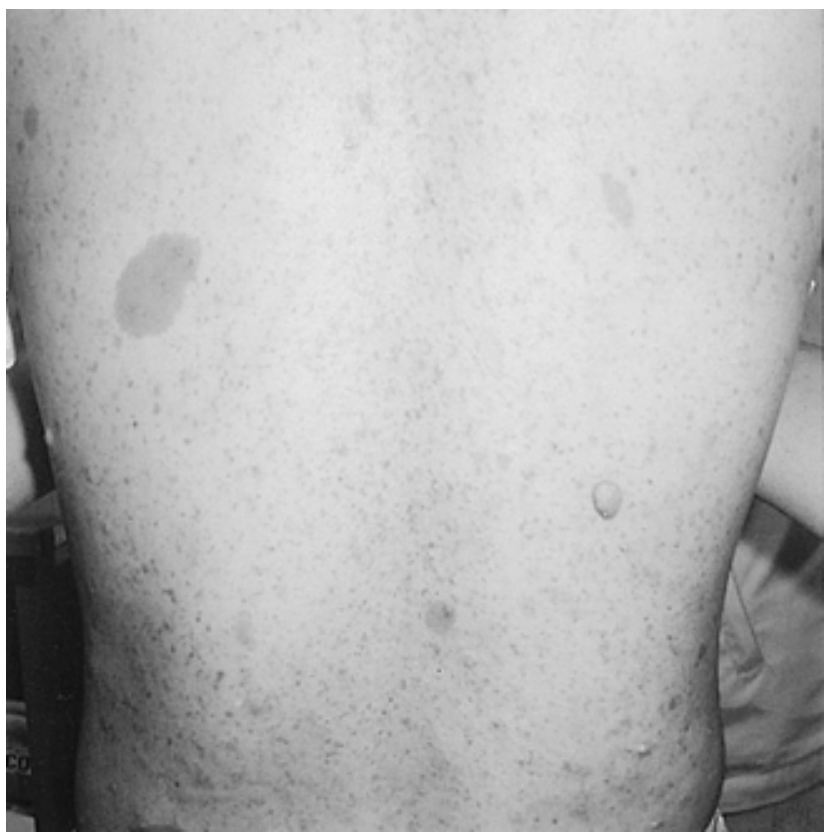

Figure 1. Skin lesions of case I. There are numerous, small subcutaneous nodules of neurofibroma and brownish-pigmented lesions (café au lait spots) in the trunk.

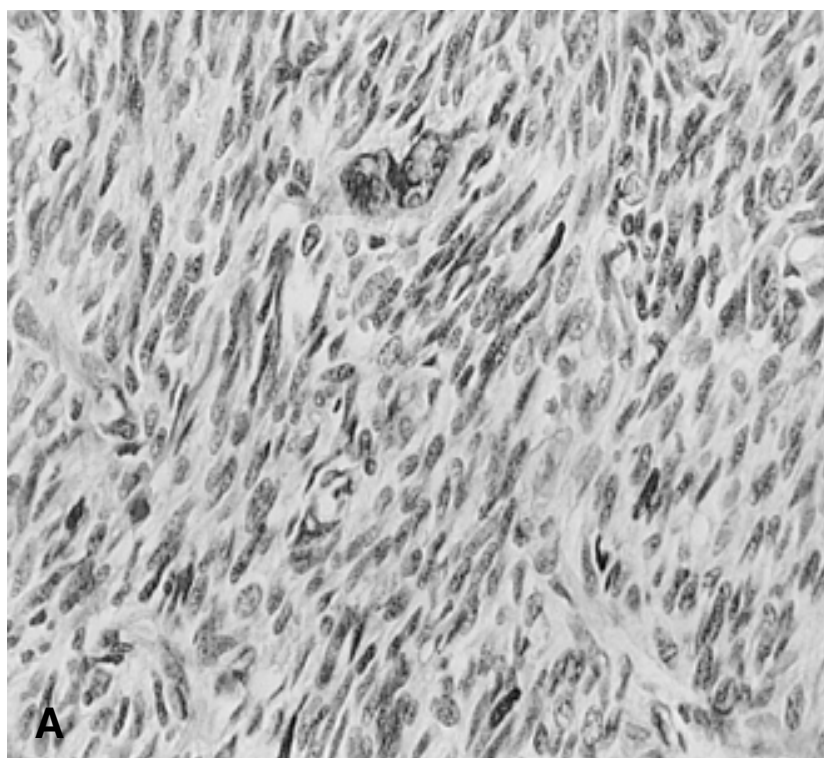

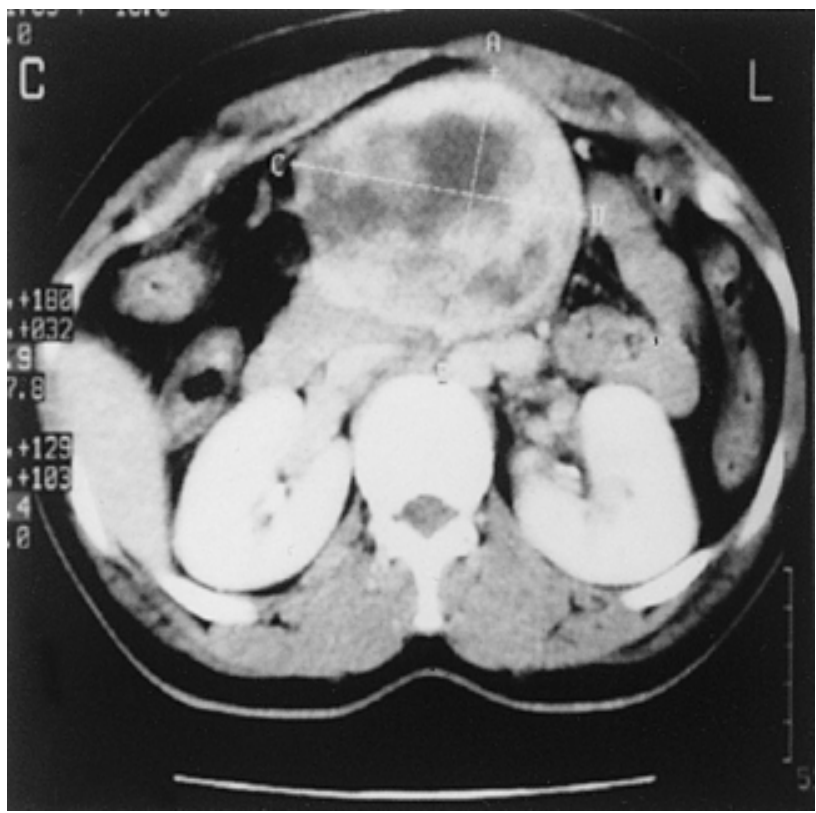

Figure 2. Abdominal CT scan of case I demonstrating a large (approximately $9 \times 10 \mathrm{~cm}$ ) inhomogeneous mass with an area of central necrosis.

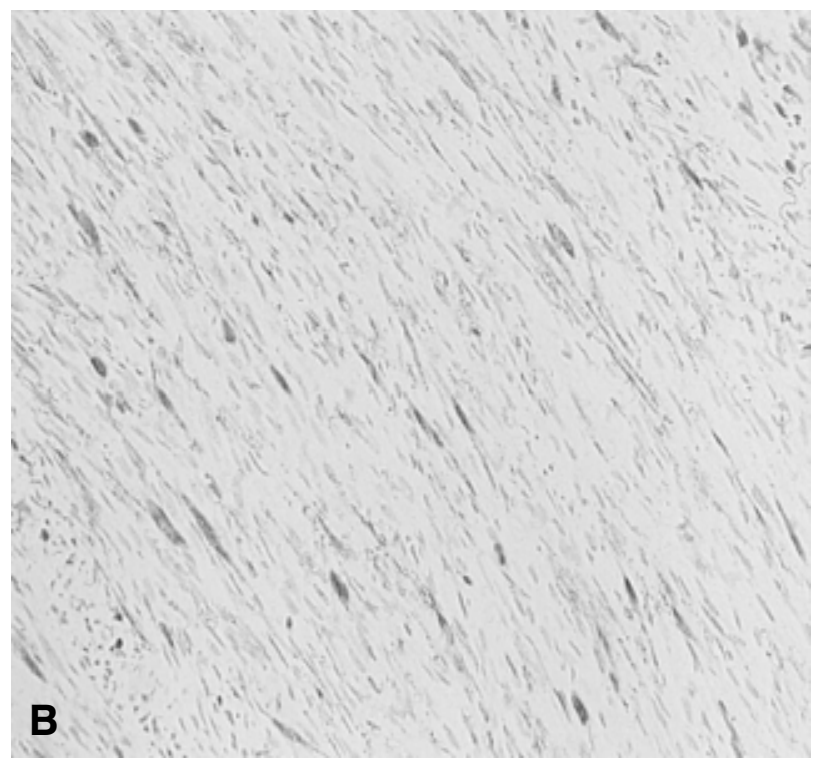

Figure 3. (A) The tumor cells show frequent mitoses of more than five per one high power field $(H \& E$ stain, $\times 400)$. (B) Immunohistochemical stain for the S-100 protein shows a positive reaction.

dacarbazine $300 \mathrm{mg} / \mathrm{m}^{2}$ for 3 days), which was followed by radiotherapy (5400 cGY). The masses began to disappear after chemoradiotherapy but the disease progressed 2 months later. She was treated with a further 6 cycles of VIP chemotherapy (etoposide $75 \mathrm{mg} / \mathrm{m}^{2}$ for 5 days, ifosfamide $1,000 \mathrm{mg} / \mathrm{m}^{2}$ for 5 days, and cisplatin $20 \mathrm{mg} / \mathrm{m}^{2}$ for 5 days), with a partial response being observed. However, she died as a result of disease progression 21 months after the initial diagnosis.

Case II : A 33 year-old woman with a known neurofibromatosis was admitted due to left upper chest pain. A chest $\mathrm{PA}$ and $\mathrm{CT}$ scan demonstrated a homogenous mass measuring $3.5 \times 2.5 \mathrm{~cm}$ at the left upper lung field with a well-demarcated 

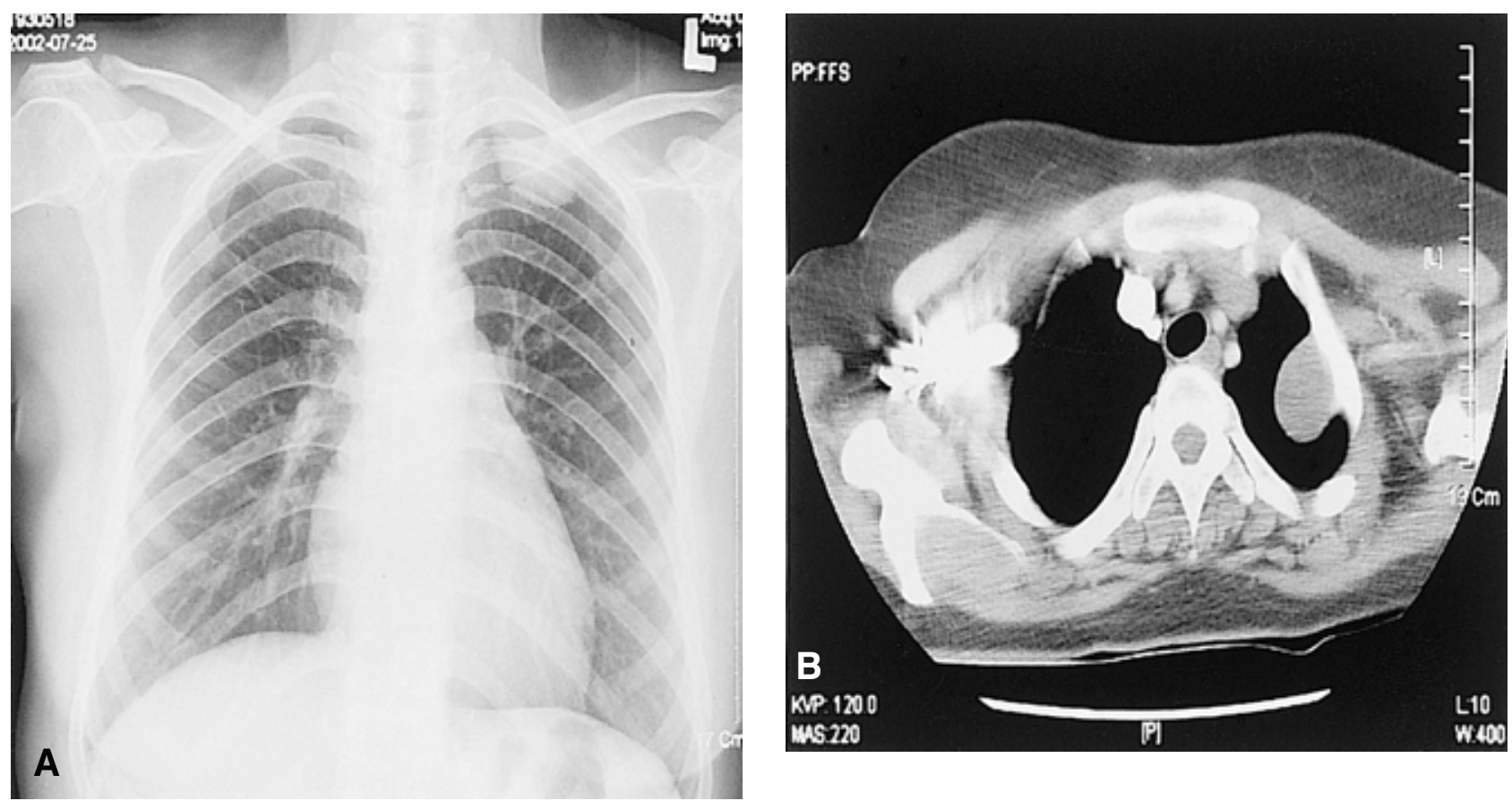

Figure 4. Chest PA (A) and CT scan (B) of case II show a homogenous mass measuring $3.5 \mathrm{~cm} \times 2.5 \mathrm{~cm}$ at the left upper lung field with a well-demarcated margin.

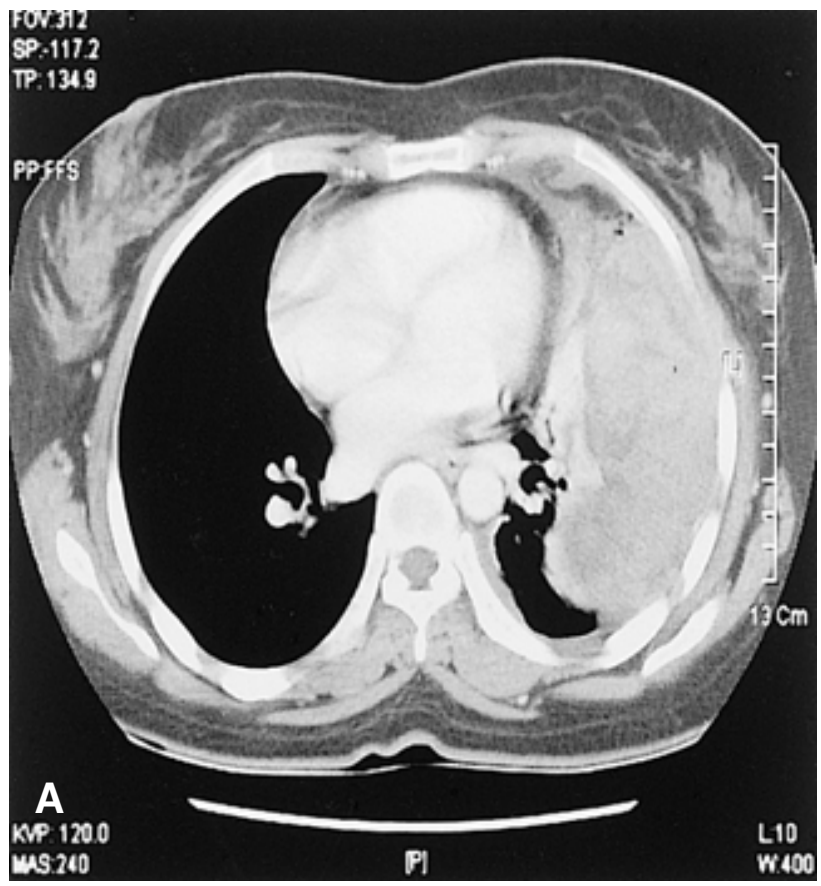

Figure 5. Chest CT scan of case II shows an inhomogeneous mass and a large amount of a left pleural effusion.

margin (Figure 4A, 4B). A wide excision was performed. The microscopic findings showed a MPNST with focal neurofibroma area and positive immunohistochemical staining for the S-100

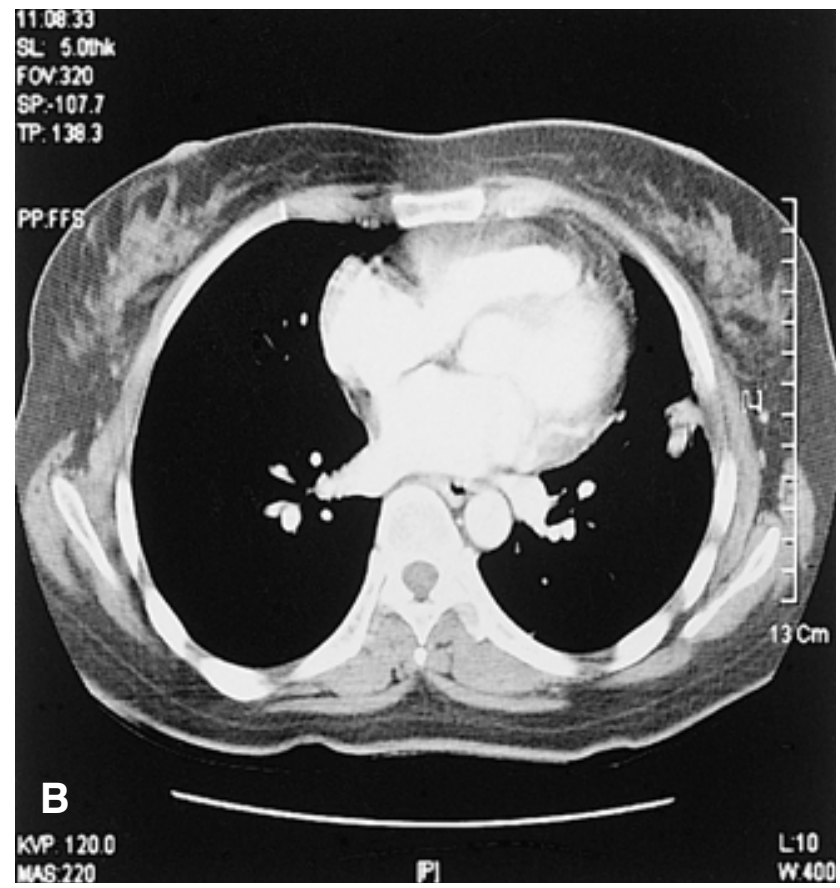

Figure 6. The chest CT scan shows that the tumor is markedly reduced after chemotherapy.

protein on the cytoplasm of the tumor cells. She was rehospitalized 6 months later due to persistent coughing and dyspnea. A chest CT scan demonstrated an inhomogeneous 
mass and a large amount of a left pleural effusion (Figure 5). A needle biopsy of the mass showed a tumor recurrence. After 6 cycles of MAID combination chemotherapy, a CT scan showed that the mass was markedly reduced (Figure 6). She is currently being treated with radiotherapy.

\section{DISCUSSION}

A neurofibromatosis type I, which is also known as von Recklinghausen's disease, is a disease of the supporting tissues of the nervous system and is one of the most common genetic disorders, affecting approximately 1 out of 3,000 live births ${ }^{4)}$. An autosomal dominant mutation at the 17q11.2 locus has been identified ${ }^{5}$. The clinical features of NFI include café au lait spots, Lisch nodules (pigmented hamartomas) of the iris, and several types of neurofibromas. Our two patients also showed multiple café au lait spots and pigmented nodular skin lesions over the trunk and extremities.

Although higher estimates appear in the literature, approximately $5 \%$ of patients with NF1 develop MPNSTs, ${ }^{2}$, which usually arises from a plexiform neurofibroma. The common anatomic locations for MPNSTs are the lower and upper extremities, the trunk, head and neck, and miscellaneous sites in descending order ${ }^{6)}$. Sordillo PP et $\mathrm{al}^{7)}$ and Guccion JG et $\mathrm{al}^{8)}$ reported that tumors associated with NF1 are located more centrally than without NF1. However, intraabdominal or intrathoracic manifestation of MPNST in patients with NF1 is quite rare $^{9-11)}$.

It appears that the presence of NF1 has a negative impact on the clinicopathological features of MPNST. A histological examination of our cases revealed high-grade features. Ducatman et $\mathrm{al}^{1)}$ also reported that the tumors associated with NF1 were more often poorly differentiated and showed higher mitotic indices. Furthermore, MPNSTs associated with NF1 appeared to be aggressive in our cases. In case I, the metastases existed at the time of surgery and an early recurrence after excision was observed in case II. Ducatman et $\mathrm{al}^{1)}$ also reported that metastases and recurrences were more common for tumors associated with NF1. In addition, the mean interval from diagnosis to the first recurrence or metastasis was shorter. According to their report, $16 \%$ of the group with NF1 survived 5 years, which was significantly lower than the $53 \%$ in the other group.

Treatments for MPNST have principally included a surgical excision with and without adjuvant radiation or chemotherapy. Survival appears to be related to the ability of a complete tumor resection. Many authors have failed to show a survival benefit with the use of adjuvant radiation therapy ${ }^{12)}$. However, Basso-Ricci et $\mathrm{al}^{13)}$ reported a $56 \%$ disease free survival using combined surgical and radiation therapy for MPNST. On the other hand, there has been considerable controversy surrounding the use of adjuvant chemotherapy in adults with soft tissue sarcomas including MPNST. Because of the small sample size of the individual reports, the Sarcoma MetaAnalysis Collaboration (SMAC) performed a meta-analysis of 1568 patients that were included in 14 randomized trials of adjuvant doxorubicin-based chemotherapy versus a control ${ }^{14)}$. Their study showed improvements in the local relapse free interval, the distant relapse free survival, and the overall recurrence free survival. Currently, doxorubicin-based adjuvant chemotherapy is recommended for patients who have had a sarcoma removed but with features predicting a high likelihood of a relapse (deep location, size $>5 \mathrm{~cm}$, high histological grade). The role of chemotherapy in the treatment of a metastatic or recurrent MPNST is poorly understood due to its rarity. Several studies have reported that MPNST should be regarded as a drug sensitive neoplasm ${ }^{15,16)}$. Doxorubicin containing combination chemotherapy had moderate activity in our cases. However, there is no clear standard of care regarding the issue of palliative chemotherapy in patients with MPNST. Therefore, future multi-center clinical trials will be needed in order to determine the role of palliative chemotherapy and to define the most active chemotherapeutic regimens.

This paper reports two cases of an intraabdominal or intrathoracic presentation of a MPNST in patients with NF1. MPNST associated with NF1 showed a highly aggressive behavior and a poor prognosis. It is recommended that a MPNST associated with NF1 be treated aggressively with multi-modalities such as a radical tumor excision with a wide margin of normal tissue followed by adjuvant chemoradiotherapy. In addition, the patients should be closely monitored for the early detection of a recurrence.

\section{REFERENCES}

1) Ducatman BS, Scheithauer BW, Piepgras DG, Reiman HM, Ilstrup DM. Malignant peripheral nerve sheath tumors: a clinicopathologic study. Cancer 57:2006-2021, 1986

2) Sorensen SA, Mulvihill JJ, Nielsen A. Long-term follow-up of von Recklinghausen neurofibromatosis. N Egnl J Med 314:1010-1015, 1986

3) Riccardi V. Von Recklinghausen neurofibromatosis. N Egnl J Med 305:1617-1627, 1981

4) Riccardi V. Neurofibromatosis: phenotype, natural history and pathogenesis. $2^{\text {nd }}$ ed. Baltimore, Johns Hopkins University press, 1992

5) Wallace MR, Marchuk DA, Anderson LB, Letcher R, Odeh HM, Saulino AM, Fountain JW, Brereton A, Nicholson J, Mitchell AL. Type I neurofibromatosis gene: identification of a large transcript disrupted in three NFI patients. Science 249:181-186, 1990 
104 The Korean Journal of Internal Medicine: Vol. 20, No. 1, March, 2005

6) D'Agostino AM, Soule EH, Miller RM. Sarcoma of peripheral nerves and somatic soft tissues associated with multiple neurofibromatosis (von Recklinghausen's disease). Cancer 16:1015-1027,1963

7) Sordillo PP, Helson L, Hajdu SI, Magill GB, Kosloff C, Golbey RB, Beattie EJ. Malignant schwannoma: clinical characteristics, survival, and response to therapy. Cancer 47:2503-2509, 1981

8) Guccion JG, Enzinger FM. Malignant schwannoma associated with von Recklinghausen's neurofibromatosis. Virchows Arch A Pathol Anat Histol 383:43-57, 1979

9) Inoue M, Mitsudomi T, Osaki T, Oyama T, Haratake J, Yasumoto K. Malignant transformation of an intrathoracic neurofibroma in von Recklinghausen's disease. Scand Cardiovasc J 32:173-175, 1998

10) Suzuki H, Yamaguchi $Y$, Kimura H, Baba M, Hiroshima K. Malignant mediastinal schwannoma associated with von Recklinghausen's disease: a resected case. Nippon Kyobu Geka Gakkai Zasshi 44:864-868, 1996

11) Park JH, Choi $\mathrm{KH}$, Lee HB, Rhee $\mathrm{YK}$, Lee $\mathrm{YC}$, Chung MJ. Intrathoracic malignant peripheral nerve sheath tumor in von
Recklinghausen's disease. Korean J Intern Med 16:201-204, 2001

12) Storm FK, Eilber FR, Mirra J, Morton DL. Neurofibrosarcoma. Cancer 45:126-129, 1980

13) Basso-Ricci S. Therapy of malignant schwannomas: usefulness of an integrated radiologic: surgical therapy. J Neurosurg Sci 33:253-257, 1989

14) Sarcoma Meta-Analysis Collaboration. Adjuvant chemotherapy for localized respectable soft-tissue sarcoma of adults: meta-analysis of individual data. Lancet 350:1647-1654, 1997

15) Goldman RL, Jones SE, Heusinkveld RS. Combination chemotherapy of metastatic malignant Schwannoma with vincristine, adriamycin, cyclophosphamide, and imidazole carboxamide: a case report. Cancer 39:1955-1958, 1977

16) Blay JY, van Glabbeke M, Verweij J, van Oosterom AT, le Cesne A, Oosterhuis JW, Judson I, Nielsen OS. Advanced soft-tissue sarcoma: a disease that is potentially curable for a subset of patients treated with chemotherapy. Eur J Cancer 39:64-69, 2003 